SAME IV

\begin{tabular}{|c|c|c|c|c|c|c|}
\hline $\begin{array}{l}\text { Période..................... } \\
\text { Durée de la période } . . . \ldots \ldots \ldots\end{array}$ & $\begin{array}{c}1 \\
17 \text { jours }\end{array}$ & $\begin{array}{c}2 \\
15 \text { jours }\end{array}$ & $\begin{array}{c}3 \\
11 \text { jours }\end{array}$ & $\begin{array}{c}4 \\
6 \text { jours }\end{array}$ & $\begin{array}{c}5 \\
8 \text { jours }\end{array}$ & $\begin{array}{c}6 \\
6 \text { jours }\end{array}$ \\
\hline $\begin{array}{r}\text { Rendement en lait à } \\
4 \%(\mathrm{~kg} .) \ldots \ldots \ldots \ldots \ldots\end{array}$ & 31,3 & 31,7 & 28,2 & 30,3 & 32,3 & 31,3 \\
\hline Poids vif (kg.) . . . . . . . . . & 611 & 608 & 606 & 605 & 608 & 606 \\
\hline $\begin{array}{l}\text { Sens de variation du poids } \\
\text { vif } \ldots \ldots \ldots \ldots \ldots \ldots \ldots\end{array}$ & 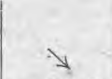 & & $\searrow$ & & $\pi$ & \\
\hline Besoins théoriques (en U. F.) & 15,2 & 15,3 & 14,2 & 15,0 & 15,6 & 15,2 \\
\hline $\begin{array}{l}\text { Ecart entre consommation } \\
\text { réelle et besoins théo- } \\
\text { riques } \ldots \ldots \ldots \ldots \ldots \ldots\end{array}$ & $+1,0$ & $+0,4$ & 0 & $+0,7$ & $+1,7$ & $+0,5$ \\
\hline
\end{tabular}

DETJE

\begin{tabular}{|c|c|c|c|c|c|c|}
\hline Période $. . . \ldots, \ldots, \ldots \ldots, \ldots$ & 1 & 2 & 3 & 4 & 5 & 6 \\
\hline Durée de la période ........... & 17 jours & 15 jours & 11 jours & 6 jours & 8 jours & 6 jours \\
\hline Rendement en lait à & & & & & & \\
\hline $\begin{array}{r}4 \% \text { (kg.) } \ldots \ldots \ldots \ldots \ldots \\
\text { Poids vif (kg.) } \ldots \ldots \ldots \ldots\end{array}$ & $\begin{array}{l}30,6 \\
696\end{array}$ & $\begin{array}{l}30,7 \\
695\end{array}$ & $\begin{array}{l}29,5 \\
689\end{array}$ & $\begin{array}{l}29,7 \\
690\end{array}$ & $\begin{array}{l}31,7 \\
693\end{array}$ & $\begin{array}{l}30,6 \\
687\end{array}$ \\
\hline $\begin{array}{l}\text { Sens de variation du poids } \\
\text { vif } \ldots \ldots \ldots \ldots \ldots \ldots \ldots\end{array}$ & $\nearrow$ & $\searrow$ & $\rightarrow$ & $\pi$ & $\pi$ & $\searrow$ \\
\hline Besoins théoriques (en U. F.) & 15,5 & 15,5 & 15,1 & 15,1 & 15,9 & 15,5 \\
\hline $\begin{array}{l}\text { Ecart entre consommation } \\
\text { réelle et besoins théo- } \\
\text { riques } \ldots \ldots \ldots \ldots \ldots \ldots\end{array}$ & $+0,6$ & $+0,1$ & $-1,0$ & $+0,5$ & $+1,3$ & $+0,1$ \\
\hline
\end{tabular}

(A suivre.)

\title{
ACIDES VOLATILS DU BEURRE PROVENANT DE VACHES ALIMENTÉES AVEC DU SON DE RIZ
}

par le Professeur Docteur Nestore MONTI

Directeur du Laboratoire Provincial de Chimie de la Ville de Pavie.

Un fermier de la province de Pavie, M. Codecà, important producteur de lait et, par conséquent, de beurre et de fromage, me soumit au mois de mars de l'année dernière un échantillon de beurre que les agents du Service sanitaire d'une grande ville voisine avaient prélevé alors qu'il livrait une fourniture de beurre de sa propre fabrication. Le beurre avait été analysé et, en raison du fort pour- 
centage de margarine qu'il contenait, le producteur avait été dénoncé à l'autorité compétente, aux fins de poursuites, conformément à la loi.

M. Codecà, en me présentant l'échantillon, me pria d'en faire l'analyse, prétendant qu'il était sûr que son beurre, provenant de la crème du lait centrifugé de ses vaches, était absolument pur. Il ajoutait que, de nombreux prélèvements de son beurre ayant été faits précédemment par les mêmes agents et celui-ci ayant toujours été trouvé d'excellente qualité, il estimait que l'échantillon qu'il me présentait devait être reconnu tel, attendu qu'il avait été obtenu avec le même lait et par la même méthode de fabrication.

Après avoir constaté que les scellés de l'échantillon étaient intacts, j'ai procédé à l'analyse du beurre, et je transcris ci-dessous les résultats obtenus :

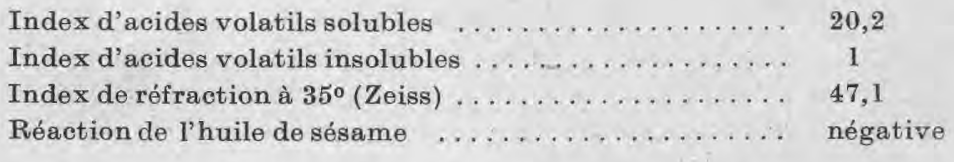

Ces résultats confirmaient le jugement émis par le laboratoire qui avait dressé la contravention.

Toutefois, le propriétaire du beurre ne fut pas persuadé et me pria d'assister à la traite des vaches de son étable, à la fabrication du beurre et de procéder ensuite à l'analyse du produit ainsi obtenu.

J'acceptai. Nous étions alors dans la deuxième quinzaine de mars. J'assistai à la traite (60 vaches), à la centrifugation du lait et à la fabrication du beurre, après avoir minutieusement examiné tous les récipients, ainsi que la baratte.

Voici les résultats obtenus à l'analyse du beurre fabriqué lors de cette expérience :

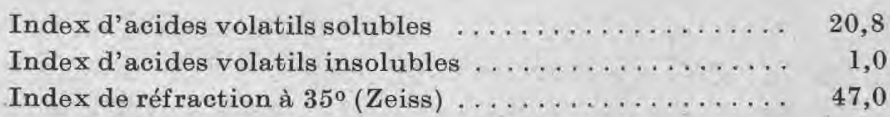

Les vaches étaient au régime sec : foin excellent, tourteau de coco, et son de riz - régime qui durait depuis un mois environ.

Ces résultats me surprirent, car je savais, d'après les nombreuses analysês que j'avais faites, que le pourcentage des acides volatils solubles des beurres de cette région est toujours très élevé $(28,5$ en moyenne) et ne descend jamais au-dessous de 27,2.

J'ai alors invité le fermier à alimenter son bétail d'après le vieux système, en éliminant le tourteau et le son de riz.

Après trois jours d'alimentation avec ce nouveau régime (herbe, 
foin, son de blé), l'analyse du beurre obtenu donnait les résultats suivants :

Index d'acides volatils solubles : 26,8 ; index d'acides volatils insolubles : 2 , après 4 jours.

Index d'acides volatils solubles : 29,2 ; index d'acides volatils insolubles :

2 , après 6 jours.

Index d'acides volatils solubles : 29,2 ; index d'acides volatils insolubles : 2 , après 12 jours.

Il est done évident que la diminution de l'index d'acides volatils solubles était due à l'alimentation, et plus précisément, au coco et au son de riz, substances qui entraient dans la composition de la ration primitive.

Comme suite à ces résultats et d'açcord avec le fermier, j'ai effectué diverses expériences pratiques sur un petit nombre de vaches (je ne les ai pas faites sur toutes, pour ne pas porter préjudice aux intérêts du producteur, qui devait, naturellement, mettre son beurre sur le marché); les expériences furent faites sur un groupe de 4 vaches (deux de race hollandaise et deux de race suisse). Voici, réunis dans la table suivante, les résultats des différentes analyses, faites, sur le beurre obtenu avec le lait de ees vaches, soumises, pendant quatre jours, à des régimes alimentaires différents :

Acides volatils Solubles Insolubles

29,2

Herbe, foin, son de blé ..............

Herbe, foin, tourteau de coeo (1 kilo-

Herbe, foin, son de riz (2 kilogrammes).

Herbe, foin, tourteau d'arachide ......

Herbe, foin, son de riz (3 kilogrammes).

Herbe, foin, son de riz (après 6 jours) ..

Herbe, foin, son de riz (après 12 jours).

29,1

25,9

28,9

26,5

19,3

16,7
Index de réfraction à $35^{\circ}$ (Zeiss)

45,6

45,0

47,0

45,8

46,1

48,5

48,6

Il résulte elairement des données ci-dessus, que la diminution des acides volatils du beurre est due au son de riz, lequel, en même temps provoque une augmentation de l'index de réfraction.

En supprimant peu à peu le son de riz de l'alimentation, on note, après 6 jours, que l'index des acides volatils et celui de la réfraction redeviennent normaux.

Cette action du son de riz est quelque peu obscure, car le son de riz est un aliment riche en protéines, en graisse et en phosphore organique (phytine), et qui donne les meilleurs résultats, aussi bien dans l'alimentation du bétail d'élevage que dans celui des vaches laitières (augmentation de production); les fabricants de beurre et de fromage ont toutefois observé à plusieurs reprises que le lait provenant de vaches alimentées avec des rations contenant du son 
de riz se travaillait mal, donnant du beurre peu consistant et des fromages ne supportant pas la maturation. Il est intéressant de noter que ces constatations faites par les fabricants, concordent entièrement avec mes expériences et les confirment.

Me basant sur le fait connu que la composition de la graisse d'un animal peut varier selon les substances grasses qu'on lui fait absorber, j'ai pensé que l'action du son de riz était due à la matière grasse qu'il contenait et $j$ 'ai alors alimenté des vaches avec du son de riz dégraissé par pression : le beurre que j'ai obtenu présentait un index d'acides volatils solubles très bas $(18,4)$. On peut par conséquent assurer que la graisse n'a aucune influence sur l'abaissement de l'index d'acidité volatile du beurre.

Les expériences se continuent en ajoutant maintenant de la graisse de riz à l'alimentation normale, de même que hous effectuons d'autres expériences pour établir à quel autre constituant est due la dite action du son de riz et pour rechercher le moyen de la neutraliser. Dès que les expériences seront terminées, je communiquerai les résultats.

J'ai cru utile de faire connaître ces observations parce qu'elles intéressent les personnes préposées à la surveillance de la production et du commeree du lait industriel ; car, si l'action du son de riz sur les acides volatils solubles peut n'avoir aucune importance pratique en ce qui concerne le lait destiné à l'alimentation, elle en a une, au contraire, très grande en ce qui concerne le lait destiné à l'industrie, attendu que les produits que l'on obtient (beurre, fromage) du lait provenant de vaches alimentées avec du son de riz, n'offrent pas les qualités de pureté requises par la loi.

\title{
LA PASTEURISATION A TEMPÉRATURE ÉLEVÉE DE COURTE DURÉE AUX ÉTATS-UNIS
}

par

\author{
M. W. YALE
}

Section de Bactériologie de la Station d'Essais Agricoles de l'Etat de New-York, Geneva (N.-Y.).

(Suite.)

\section{REVUE BIBLIOGRAPHIQUE \\ VOLUME DE LA COUCHE DE CREME}

L'idée du consommateur, que l'épaisseur de la couche de crème dans une bouteille de lait est une indication de la richesse du lait, a amené le vendeur de lait à appliquer des méthodes qui donneront un volume maximum à la couche de crème. De telles méthodes sont 